\title{
Why is there a modifying effect of gestational age on risk factors for cerebral palsy?
}

\author{
C Greenwood, P Yudkin, S Sellers, L Impey, P Doyle
}

Arch Dis Child Fetal Neonatal Ed 2005;90:F141-F146. doi: 10.1136/adc.2004.052860

See end of article for authors' affiliations

......................

Correspondence to: Dr Greenwood, Level 4, The Women's Centre, John Radcliffe Hospital, Headley Way, Headington, Oxford OX3 9DU, UK:

Catherine.Greenwood@ orh.nhs.uk

Accepted

16 September 2004

\begin{abstract}
Objective: To investigate risk factors for cerebral palsy in relation to gestational age.
Design: Three case-control studies within a geographically defined cohort.

Setting: The former Oxfordshire Health Authority.

Participants: A total of 235 singleton children with cerebral palsy not of postnatal origin, born between 1984 and 1993, identified from the Oxford Register of Early Childhood Impairment; 646 controls matched for gestation in three bands: $\leqslant 32$ weeks; 33-36 weeks; $\geqslant 37$ weeks.

Results: Markers of intrapartum hypoxia and infection were associated with an increased risk of cerebral palsy in term and preterm infants. The odds ratio (OR) for hypoxia was 12.2 (95\% confidence interval 1.2 to 119$)$ at $\leqslant 32$ weeks and $146(7.4$ to 3651$)$ at $\geqslant 37$ weeks. Corresponding ORs for neonatal sepsis were 3.1 (1.8 to 5.4) and 10.6 (2.1 to 51.9). In contrast, pre-eclampsia carried an increased risk of cerebral palsy at $\geqslant 37$ weeks (OR 5.1 (2.2 to 12.0)) but a decreased risk at $\leqslant 32$ weeks (OR 0.4 (0.2 to 1.0)). However, all infants $\leqslant 32$ weeks with maternal pre-eclampsia were delivered electively, and their risk of cerebral palsy was no lower than that of other electively delivered $\leqslant 32$ week infants (OR 0.9 (0.3 to 2.7$)$ ). Nearly $60 \%$ of $\leqslant 32$ week controls were delivered after spontaneous preterm labour, itself an abnormal event.

Conclusion: Inflammatory processes, including pre-eclampsia, are important in the aetiology of cerebral palsy. The apparent reduced risk of cerebral palsy associated with pre-eclampsia in very preterm infants is driven by the characteristics of the gestation matched control group. Use of the term "protective" in this context should be abandoned.
\end{abstract}

\section{METHODS}

Subjects

Singletons born to mothers resident in Oxfordshire or West Berkshire health districts 1984-1993 inclusive were studied. The data were drawn from three sources: two previous casecontrol studies (studies 1 and 2$)^{29}$ and the present study (study 3). Study 1 investigated babies born at term ( $\geqslant 37$ weeks gestation) between 1984 and 1987. ${ }^{9}$ Study 2 examined risk factors in babies born at or before 32 weeks gestation $^{2}{ }^{9}$ between 1984 and 1990. Data on antenatal and intrapartum risk factors were collected in both. Further data were collected in the present study (study 3 ) to complete the data set on all cases and controls born between 1984 and 1993 and at all gestational ages, to mothers resident in Oxfordshire or West Berkshire. Gestational age was estimated using menstrual dates, unless they were uncertain or the ultrasound estimation of dates was more than two weeks different, in which cases ultrasound dates were preferred.

\section{Cases}

Cases of CP were identified from the Oxford Register of Early Childhood Impairment. ${ }^{10}$ The definition of CP used by the register is that of a permanent impairment of voluntary movement or posture presumed to be due to damage to the immature brain. The register is compiled from many sources, and the condition of the child is assessed at 3 and 5 years of age. In general, children are not included as "cases" on the register until the age of 5 years, although children with clear signs of $\mathrm{CP}$ who die between the age of 1 and 5 years are also included. Babies for whom the cause of CP was either genetic or a fetal malformation syndrome and those who had a

Abbreviations: $\mathrm{CP}$, cerebral palsy; SGA, small for gestational age why is there an apparent modifying effect of gestational age on risk factors for $\mathrm{CP}$ ? 
Table 1 Cases and controls, stratified by gestational age band

\begin{tabular}{|c|c|c|c|c|c|c|}
\hline & \multicolumn{2}{|c|}{$\leqslant 32$ weeks } & \multicolumn{2}{|c|}{$33-36$ weeks } & \multicolumn{2}{|c|}{$\geqslant 37$ weeks } \\
\hline & Cases & Controls & Cases & Controls & Cases & Controls \\
\hline Eligible & 75 & 291 & 41 & 82 & 161 & 286 \\
\hline Missing records* & 0 & 6 & 0 & 6 & 4 & 1 \\
\hline $\begin{array}{l}\text { Excluded (developmental } \\
\text { brain lesion) }\end{array}$ & 2 & 0 & 5 & - & 31 & \\
\hline $\begin{array}{l}\text { Reallocation of controls to } \\
\text { correct gestational age band }\end{array}$ & 0 & 1 added & 0 & 6 removed & 0 & 5 added \\
\hline Final number included in study & 73 & 286 & 36 & 70 & 126 & 290 \\
\hline
\end{tabular}

clearly identified hypoxic event after 28 days of life were excluded from this study.

\section{Controls}

Study $1: \geqslant 37$ weeks gestation ${ }^{9}$

Two controls were drawn for each case. The two singleton term deliveries before the index case were identified from the relevant hospital birth register.

Study 2: 32 weeks gestation or less ${ }^{2} 10$

Four controls per case (236) were randomly selected from 474 eligible very preterm infants $(\leqslant 32$ completed weeks gestation) born in Oxfordshire or West Berkshire who survived until discharge and did not develop CP

\section{Study 3: all gestations}

Gestational age was stratified into three age bands: $\leqslant 32$ weeks, $33-36$ weeks, and $\geqslant 37$ weeks gestation. Two controls were identified from the hospital birth register including the index case. The two singleton deliveries of the same gestational age band as the index case, and immediately preceding it, were selected.

Six controls identified for the middle gestational age band (33-36 weeks) were found to lie outside this band when the case notes were reviewed (table 1 ).

\section{Data sources}

In all three studies an obstetrician blind to the outcome of the children reviewed the obstetric and neonatal records of the subjects. All studies included data on demography, maternal medical history, family history, obstetric history, antenatal history, labour details including intrapartum monitoring, placental histology, and neonatal history. Before amalgamation of the data, the definitions used for each variable were compared to ensure that they were equivalent. Only variables with identical definitions in all three studies were included in this analysis.

\section{Definitions}

The following definitions were used:

- Pre-eclampsia: Maternal diastolic blood pressure $\geqslant 90 \mathrm{~mm}$ $\mathrm{Hg}$ on two or more occasions with proteinuria $\mathrm{I}+$ or more on ward testing or $>0.5 \mathrm{~g} / 24$ hours on laboratory testing.

- Elective caesarean section: Caesarean section performed before the onset of labour.

- Elective delivery: Delivery by any route where there was no spontaneous onset of labour-that is, birth was after induction of labour or elective caesarean.

- Abruption: A significant antepartum haemorrhage after 20 weeks gestation or intrapartum haemorrhage with an associated clinical diagnosis of abruption, usually based on uterine tenderness or irritability or a retroplacental clot.
- Pre-labour rupture of membranes: Rupture of the fetal membranes at any time before the onset of regular painful contractions.

- Chorioamnionitis: Either a clinical diagnosis based on either two out of three of maternal temperature $\geqslant 38^{\circ} \mathrm{C} /$ uterine tenderness/foul smelling vaginal discharge or histological chorioamnionitis.

- Intrapartum pyrexia: Maternal temperature of $\geqslant 38^{\circ} \mathrm{C}$ in labour on two occasions.

- Small for gestational age (SGA): Measurements based on serial ultrasound show that babies born early tend to be smaller than babies of the same gestation who continue to term. ${ }^{11}$ Thus many of the available nomograms of birth weight based on liveborn children underestimate the proportion of SGA babies in the preterm population. In addition, owing to small numbers, centiles based on delivered populations are less accurate at the extremes. Therefore inadequate intrauterine growth was measured using a method modified from Blair and Stanley. ${ }^{12}$ The ratio of attained birth weight to expected birth weight was calculated for each infant taking gestational age and sex into account, by dividing the actual birth weight by the expected (mean) birth weight derived from nomograms in use at the John Radcliffe Hospital. ${ }^{13}$ The mean (SD) of this ratio for the control population was $1.03(0.13)$. The values obtained are similar to those reported by Blair and Stanley. ${ }^{12}$ Babies were then defined as SGA if the birthweight ratio was $>$ ISD below the mean for the control population (ratio $<0.90$ ).

- Acute intrapartum hypoxic event: Either umbilical arterial $\mathrm{pH}$ at birth of less than 7.00 or not measured with both early onset neonatal seizures during the first day of life and a five minute Apgar score of less than 7.14

- Neonatal sepsis: Either clinical diagnosis or positive microbiological culture from blood or cerebrospinal fluid within the first 28 days of life.

\section{Statistical methods}

Data analysis was performed using SPSS (Chicago, Illinois, USA; version 10). Odds ratios (ORs) and 95\% confidence intervals were calculated using logistic regression. All ORs were adjusted for the source of the data-that is, study 1,2 , or 3-and additional adjustments were made for the analyses presented in table 4 (variables used for adjustment shown in footnotes of table 4). ORs were regarded as statistically significant if the 95\% confidence interval excluded 1.00 . However, the large number of ORs calculated should be remembered in interpreting the results.

\section{RESULTS}

In $38 \mathrm{CP}$ cases, the cause was genetic or a fetal malformation syndrome, thus 239/277 children were eligible, of whom 235 


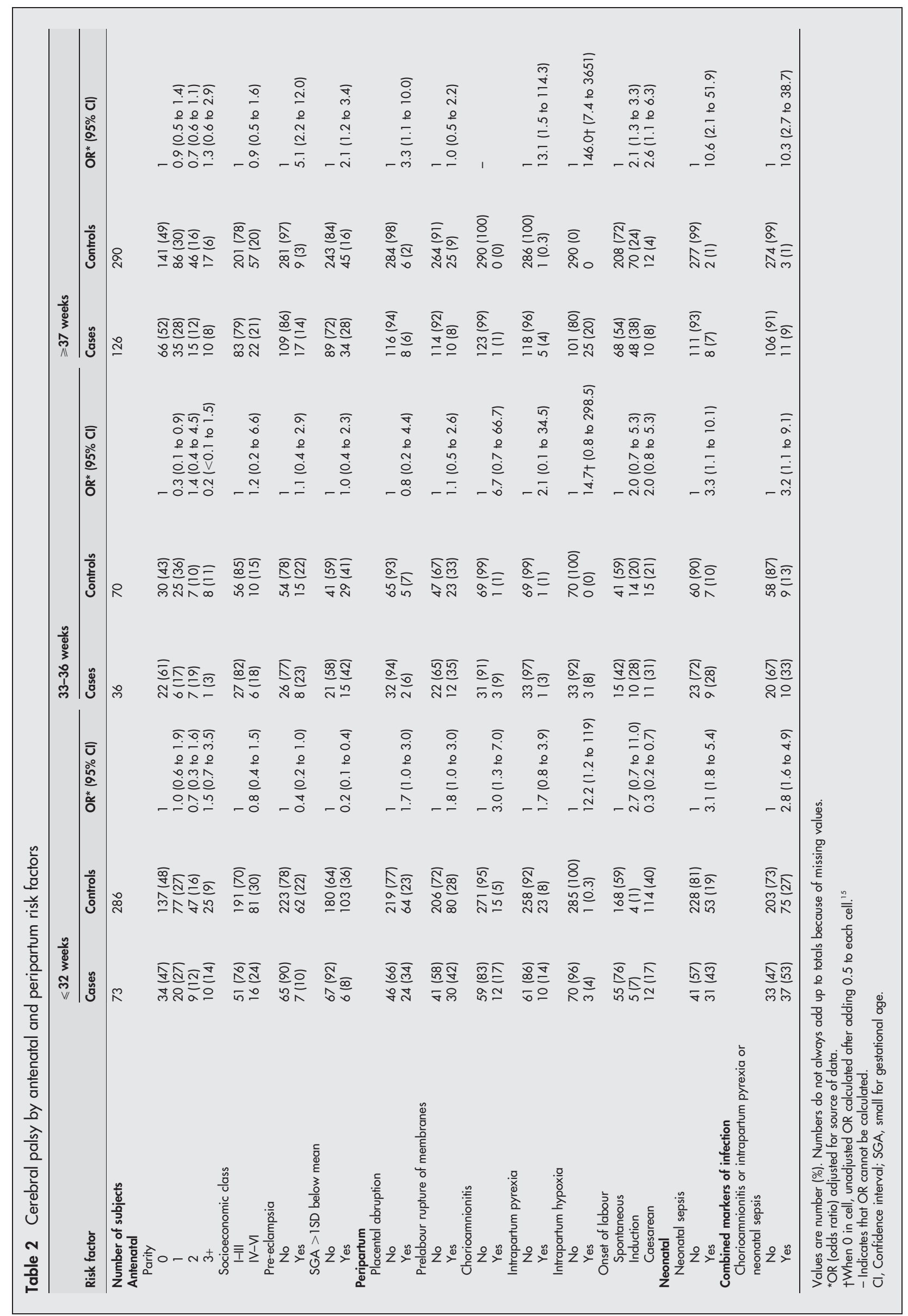


(98\%) were included in this study (case notes missing for four children). Table 1 shows the distribution of cases across the gestational age range. Almost a third $(73 / 235 ; 31 \%)$ were born at 32 weeks gestation or less. Over a half (126/235: 54\%) were born at term, leaving a small group of $36(15 \%)$ born between 33 and 36 weeks. The mean gestational age in weeks of cases and controls within each stratum was: $\leqslant 32$ weeks, cases 28.6, controls 29.9; 33-36 weeks, cases 34.4, controls 34.9; $\geqslant 37$ weeks, cases 39.4, controls 39.6.

Based on population denominator data held by the register, the rate of CP per 1000 live births in each gestational age stratum was $41.8 / 1000$ live births at $\leqslant 32$ weeks, 5.9/1000 live births at 33-36 weeks, and 1.0/1000 live births at $\geqslant 37$ weeks.

Table 2 shows the univariate analysis of the relation of antenatal, peripartum, and neonatal factors and CP.

\section{Antenatal factors}

Parity and socioeconomic status were not associated with CP. Pre-eclampsia was common in very preterm ( $\leqslant 32$ weeks) controls $(22 \%)$ but not in term controls $(3 \%)$. There was a significantly increased risk of CP with pre-eclampsia in term babies (OR 5.1, 95\% CI 2.2 tol2.0), but no evidence of an association in babies of 33-36 weeks gestation (OR 1.1, 0.4 to 2.9). Among babies born extremely preterm, pre-eclampsia was associated with a reduced incidence of CP (OR 0.4, 0.2 to 1.0). Few mothers during the period of this study were given antenatal steroids to induce fetal lung maturity (9/73 cases (one of whom had pre-eclampsia) and 27/283 controls (five with pre-eclampsia); the reduced risk of CP remained after adjustment for their use (AOR 0.4 (0.2 to 0.9)).

There was a high proportion of babies who were SGA (birthweight ratio $>$ ISD below the mean) among both the $\leqslant 32$ week controls $(103 / 283 ; 36 \%)$ and the 33-36 week controls $(29 / 70 ; 41 \%)$. The risk of CP associated with being SGA increased across the gestational age strata. For babies born at or below 32 weeks gestation, there was a strongly reduced risk of CP associated with SGA (OR 0.2: 95\% CI 0.1 to $0.4)$, for babies born at 33-36 weeks gestation, the OR was 1.0 (0.4 to 2.3), and among term babies there was a doubling of the risk of CP (OR 2.1, 1.2 to 3.4).

\section{Peripartum factors}

Placental abruption, prelabour rupture of membranes, chorioamnionitis, and intrapartum pyrexia were all more common in very preterm $(\leqslant 32$ week) controls than in term controls. Placental abruption was associated with an increased risk of CP in both very preterm and term groups although not in the 33-36 week group. Prelabour rupture of membranes was associated with an increased risk of $\mathrm{CP}$ in the very preterm group (OR1.8, 1.0 to 3.0), but there was no association in the other two gestational age strata. In the very preterm group, chorioamnionitis was clearly associated with an increased risk of $\mathrm{CP}$ (OR 3.0, 1.3-7.0); it was more common in cases than controls in the other gestational age strata, but numbers were too small for precise estimates of risk. Intrapartum pyrexia, however, was associated with $\mathrm{CP}$ in the term group (OR 13.1, 1.5 to 114.3). Intrapartum hypoxia occurred in only one very preterm control and not at all in the other two control groups. Although numbers were small, it appeared to be a strong risk factor for CP in all gestational age groups.

The pattern of delivery differed in the three gestational age groups. Induction of labour occurred in $24 \%$ of term controls, $20 \%$ of the 33-36 week controls, and only $1 \%$ of the $\leqslant 32$ week group, whereas caesarean section before labour occurred in $40 \%$ of controls $\leqslant 32$ weeks compared with $21.4 \%$ of controls 33-36 weeks and 4\% of term controls. Induction was more common in cases than controls in all strata, but the association with $\mathrm{CP}$ was significant only in the term group (OR 2.1, 1.3 to 3.3). However, among babies in the $\leqslant 32$ weeks group, prelabour caesarean section was associated with a reduced risk of CP (OR 0.3, 0.2 to 0.7 ) whereas among those born at term, there was an increased risk (OR $2.6,1.1$ to 6.3 ).

\section{Neonatal factors}

Neonatal sepsis was a strong risk factor for $\mathrm{CP}$ in all gestational age groups.

\section{Combined markers of infection}

Using a combined variable of chorioamnionitis and/or intrapartum pyrexia and/or neonatal sepsis, there was a strong association between evidence of infection and CP in all the gestational age groups ( $\leqslant 32$ weeks, OR 2.8, 1.6 to 4.9 ; 33-36 weeks, OR 3.2, 1.1 to 9.1; term, OR 10.3, 2.7 to 38.7).

On univariate analysis therefore placental abruption, intrapartum hypoxia, and infection appear to be associated with an increased risk of CP in both the very preterm and term gestational age groups. However, gestational age appears to modify the association between $\mathrm{CP}$ and preeclampsia, SGA, and elective delivery. These factors are associated with an increased risk of CP in term babies but are associated with a lower risk of CP in babies born at less than 33 weeks gestation.

To explain this finding, we examined the characteristics of the gestation matched control group in the very preterm stratum (table 3 ). We identified two main groups of preterm births: the first occurred after elective delivery, triggered largely by pre-eclampsia and/or SGA; the second occurred after spontaneous onset of preterm labour, triggered by infection, SGA, and other, unknown, causes. We might therefore regard the factors that predispose to preterm delivery-particularly pre-eclampsia, SGA, and infectionas the underlying risk factors, whereas the mode of delivery

Table 3 Rate of pre-eclampsia, small for gestational age (SGA), and markers of infection in control infants $\leqslant 32$ weeks gestation, stratified by spontaneous and elective delivery

\begin{tabular}{|c|c|c|c|c|}
\hline & \multicolumn{2}{|c|}{ Spontaneous delivery $(n=168)$} & \multicolumn{2}{|c|}{ Elective delivery $(n=118)$} \\
\hline & $\mathbf{n}$ & $\%$ & n & $\%$ \\
\hline Pre-eclampsia alone & 0 & 0 & 13 & 11 \\
\hline Pre-eclampsia+SGA & 0 & 0 & 35 & 30 \\
\hline Pre-eclampsia+infection & 0 & 0 & 3 & 3 \\
\hline Pre-eclampsia+SGA+infection & 0 & 0 & 11 & 10 \\
\hline SGA alone & 32 & 20 & 14 & 12 \\
\hline SGA+infection & 4 & 2 & 5 & 4 \\
\hline Markers of infection alone & 38 & 23 & 14 & 12 \\
\hline None of these markers & 88 & 54 & 20 & 17 \\
\hline
\end{tabular}

Data are incomplete for six infants born by spontaneous delivery and three born by elective delivery. Percentages are based on numbers with complete data. 


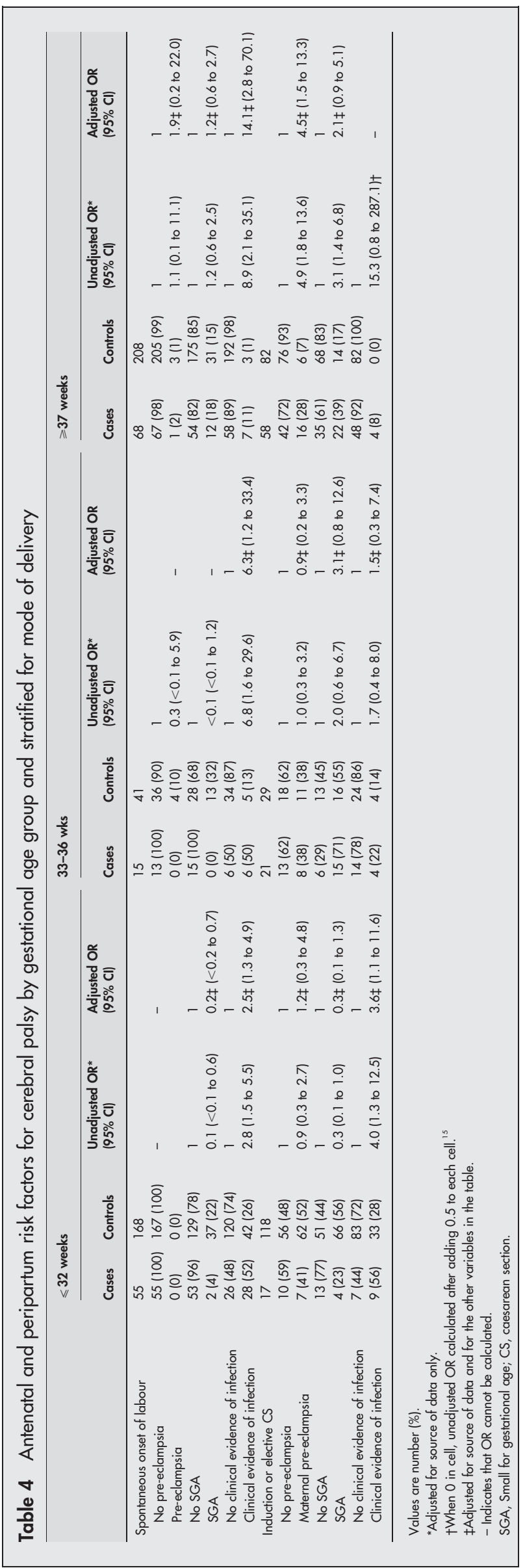

(elective or spontaneous) is merely part of a causal pathway. To clarify the role of these risk factors, we performed an analysis stratified by mode of delivery (table 4).

\section{Risk factors for CP stratified by mode of delivery Preterm group}

Among those delivered electively, pre-eclampsia was no longer associated with a reduced risk of CP (OR 0.9, 0.3 to 2.7), even after adjustment for SGA and infection. SGA, however, was associated with a reduced risk of $\mathrm{CP}$ in both the spontaneously and electively delivered strata, and this association was broadly unchanged after adjustment for pre-eclampsia and the presence of a marker of infection. On the other hand, infection retained a strong positive association with CP in preterm infants whether delivered spontaneously or electively, and after adjustment for preeclampsia and being SGA.

\section{Term group}

Pre-eclampsia in the term group remained a risk factor in the electively delivered group even after adjustment for SGA and infection but not in the spontaneously delivered group. Similarly SGA was associated with an increased risk of CP in the electively delivered group but not those who laboured spontaneously. This association was weakened after adjustment for pre-eclampsia and infection. Infection appeared to be a risk factor in term babies in both strata.

\section{DISCUSSION}

This paper examines the inter-relationship of antenatal and intrapartum risk factors for CP in a complete geographically defined cohort of singletons born at all gestations.

Gestational age appears to modify the effect of risk factors for CP, particularly pre-eclampsia and SGA; these appear protective before 33 weeks gestation, but are associated with an apparent increased risk of CP in term babies. Several explanations have previously been put forward to explain this counterintuitive phenomenon. Firstly, babies with cerebral damage delivered very preterm to mothers with preeclampsia may be particularly likely to die. With current management, however, most babies delivered preterm because of severe pre-eclampsia survive. ${ }^{16}$ Secondly, magnesium sulphate, used in many centres for the prevention or treatment of eclamptic seizures, may be neuroprotective. ${ }^{17}$ This has been examined in trials. ${ }^{18}{ }^{19}$ Our data refute this because the effect remains despite magnesium not being used in any of the pregnancies contributing to this study. Thirdly, pre-eclampsia may be protective because of treatment regimens such as antenatal steroids and elective caesarean section. With regard to the latter point, our data confirm that pre-eclamptic women are indeed usually delivered electively but that the "sparing" effect is independent of steroid usage. The importance of the distinct pathways leading to preterm delivery after pre-eclampsia and after spontaneous preterm labour is discussed below.

An alternative explanation of the negative association of $\mathrm{CP}$ and pre-eclampsia and SGA in the preterm population lies in the methodology of the case-control study, specifically, in the characteristics of the controls matched for gestational age. Preterm birth is an abnormal event and all pathways that lead to preterm birth are pathological. Our data confirm this: adverse antenatal and intrapartum events are much more common in our preterm control groups than among our term controls. Therefore babies born very preterm do not represent the distribution of characteristics in the source population of the cases-that is, all those delivered and undelivered at that gestation-which is, of course, the role of controls in a case-control study. This, we suggest, is the main 
driver for the apparent "protective" effects observed in our study.

The apparent negative association between $\mathrm{CP}$ and preeclampsia in infants born at $\leqslant 32$ weeks may be explored further by considering the two distinct pathways to preterm birth separately. In none of the preterm cases with maternal pre-eclampsia did spontaneous labour occur; all pre-eclamptic mothers were delivered electively after medical intervention. It is therefore appropriate to compare them with controls delivered electively at this gestation. This comparison does not show pre-eclampsia to be protective. The odds ratio for pre-eclampsia is 1.2 , with a wide confidence interval compatible with either a positive or a negative association between pre-eclampsia and CP. Because no infants born at $\leqslant 32$ weeks to pre-eclamptic mothers were delivered after spontaneous onset of labour, the effect of pre-eclampsia under these circumstances cannot be calculated.

SGA, like pre-eclampsia, produced some interesting findings. However, in contrast with pre-eclampsia, SGA was associated with a reduced odds ratio for CP among both electively and spontaneously delivered preterm infants. There are a number of possible explanations for this. Firstly, some distortion of the odds ratio could have been produced by our use of neonatal rather than fetal growth standards..$^{20}$ Secondly, it is possible that the mechanism initiating spontaneous labour in the presence of a chronically stressed fetus that is SGA may be an adaptive response, a process that carries less risk of white matter damage than an infective mechanism. ${ }^{21}$ Thirdly, if CP is itself a cause of spontaneous preterm birth, then other risk factors independently associated with spontaneous preterm birth, such as SGA, may appear to be protective. However, the different result for SGA and pre-eclampsia in elective preterm deliveries is puzzling. Further work is needed to clarify the causal pathways involved in preterm birth, and to identify sources of bias in epidemiological studies of CP risk in preterm babies. The best approach will be large scale prospective cohort studies of pregnancy.

In conclusion, we suggest that the so called protective effect of maternal pre-eclampsia and being SGA among very preterm infants can largely be explained by characteristics of the gestation matched control group. The term "protective" should be abandoned in this context. Notwithstanding these findings, the increased risk of CP among preterm infants, whatever the cause of preterm birth, needs to be recognised.

\section{ACKNOWLEDGEMENTS}

We are grateful to Ann Johnson, formally of the National Perinatal Epidemiology Unit, for her work in conceiving, designing, and analysing this study, and in help with earlier drafting of the article, as well as running the ORECI database.

\section{Authors' affiliations}

C Greenwood, National Perinatal Epidemiology Unit, Old Road, Headington, Oxford OX3 7LF, UK and John Raddliffe Hospital, Headley Way, Oxford OX3 9DU, UK
P Yudkin, Department of Primary Health Care, Old Road, Headington, Oxford OX3 7LF, UK

S Sellers, United Bristol Hospital Trust, formerly John Radcliffe Hospital, Headley Way, Oxford OX3 9DU, UK

L Impey, John Radcliffe Hospital, Headley Way, Oxford OX3 9DU, UK P Doyle, London School of Hygiene and Tropical Diseases, Keppel St, London $\mathrm{WCl}$, UK

Financial support: The study was partly supported by a grant from NHS Executive, South East Region Research and Development.

Competing interests: none declared

\section{REFERENCES}

1 O'Shea TM, Dammann O. Antecedents of cerebral palsy in very low-birth weight infants. Clin Perinatol 2000;27:285-302.

2 Murphy DJ, Sellers S, MacKenzie IZ, et al. Case-control study of antenatal and intrapartum risk factors for cerebral palsy in very preterm singleton babies. Lancet 1995;346: 1449-54.

3 Grether JK, Nelson KB. Maternal infection and cerebral palsy in infants of normal birth weight. JAMA 1997;278:207-11.

4 Wu YW, Escobar GJ, Grether JK, et al. Chorioamnionitis and cerebral palsy in term and near-term infants. JAMA 2003;290:2677.

5 Impey L, Greenwood C, MacQuillan K, et al. Fever in labour and neonatal encephalopathy: a prospective cohort study. BJOG 2001;108:594-7.

6 Redman CW, Sacks GP, Sargent IL. Preeclampsia: an excessive maternal inflammatory response to pregnancy. Am J Obstet Gynecol 1999; 180:499-506

7 Davidge ST, Signorella AP, Lykins DL, et al. Evidence of endothelial activation and endothelial activators in cord blood of infants of pre-eclamptic women. Am J Obstet Gynecol 1996;175:1301-6.

8 Impey L, Greenwood C, Sheil O, et al. The relationship between preeclampsia at term and neonatal encephalopathy. Arch Dis Child Fetal Neonatal Ed 2001;85:F170-2.

9 Gaffney G, Sellers S, Flavell V, et al. Case-control study of intrapartum care, cerebral palsy, and perinatal death. BMJ 1994;308:743-50.

10 Johnson A, King R. A regional register of early childhood impairments: a discussion paper. The Steering Committee of the Oxford Region Child Development Project. Community Med 1989;11:352-63.

11 Marsal K. Antenatal diagnosis of intrauterine growth restriction by ultrasound. Int J Technol Assess Health Care 1992;8(suppl 1):160-9.

12 Blair E, Stanley F. Intrauterine growth and spastic cerebral palsy. I. Association with gestational age. Am J Obstet Gynecol 1990;162:229-37.

13 Yudkin PL, Aboualfa M, Eyre JA, et al. New birthweight and head circumference centiles for gestational ages 24 to 42 weeks. Early Hum Dev 1987; 15:45-52.

14 MacLennan A. A template for defining causal relation between acute intrapartum events and cerebral palsy: international consensus statement. BMJ 1999;319:1054-9.

15 Walter SD. Small sample estimation of log odds ratios from logistic regression and fourfold tables. Stat Med 1985:4:437-44.

16 Murphy DJ, Stirrat GM. Mortality and morbidity associated with early onset pre-eclampsia. Hypertens Pregnancy 2000;19:221-13.

17 Hirtz DG, Nelson K. Magnesium sulphate and cerebral palsy in premature infants. Curr Opin Pediatr 1998;10:131-7.

18 Mittendorf R, Dambrosia J, Pryde PG, et al. Association between the use of antenatal magnesium sulfate in preterm labor and adverse health outcomes in infants. Am J Obstet Gynecol 2002;186:1111-18.

19 Crowther CA, Hiller JE, Doyle LW, et al. Australasian Collaborative Trial of Magnesium Sulphate (ACTOMg SO4). Effect of magnesium sulfate given for neuroprotection before preterm birth: a randomized controlled trial. JAMA 2003;290:2669-76.

20 Jarvis S, Glinianaia SV, Torrioli MG, et al. Surveillance of Cerebral Palsy in Europe (SCPE) collaboration of European Cerebral Palsy Registers. Cerebral palsy and intrauterine growth in single births: European collaborative study. Lancet 2003:362:1106-11.

21 Zeitlin J, Ancel PY, Saurel-Cubizolles MJ, et al. The relationship between intrauterine growth restriction and preterm delivery: an empirical approach using data from a European case-control study. BJOG 2000;107:750-8. 